\title{
Delayed False Elevation of Circulating Tacrolimus Concentrations after Cord Blood Transplantation in a Patient with Myelodysplastic Syndrome
}

\author{
Kiyohito Hayashi ${ }^{1}$, Taizo Tasaka ${ }^{1}$, Tadashi Hirose ${ }^{1}$, Satoko Furukawa ${ }^{2}$, Katsunori Kohguchi ${ }^{2}$, \\ Yoshiko Matsuhashi ${ }^{1}$, Hideho Wada ${ }^{1}$, Kaoru Tohyama ${ }^{2}$ and Takashi Sugihara ${ }^{1}$
}

\begin{abstract}
We herein describe the case of a 60-year-old man with a history of Behçet's disease and myelodysplastic syndrome who received cord blood transplantation (CBT). The patient was given anti-thymocyte globulin conditioning and tacrolimus to prevent graft-versus-host disease. Two months after CBT, his blood Tac concentration measured by an antibody-conjugated magnetic immunoassay (ACMIA) was found to have increased $>4$-fold, even after the Tac treatment was stopped. This false response was caused by the interference of endogenous heterophilic antibodies with ACMIA. Therefore, physicians must be aware of possible false ACMIA results for patients with a history of autoimmune disease and/or treated by xenogeneic antibody therapy.
\end{abstract}

Key words: antibody-conjugated magnetic immunoassay, cord blood transplantation, tacrolimus, autoimmune diseases

(Intern Med 53: 2635-2638, 2014)

(DOI: 10.2169/internalmedicine.53.2170)

\section{Introduction}

Tacrolimus (Tac), a compound secreted by Streptomyces tsukubaensis, is known to act as a potent immunosuppressant on T lymphocytes. Accordingly, Tac is commonly used for the prophylaxis of graft-versus-host disease (GVHD) during hematopoietic stem cell transplantation (HSCT). However, the therapeutic window of Tac is very narrow. A suboptimal dose can result either in potential graft rejection or GVHD. Conversely, an overdose can cause side effects (i.e., renal insufficiency, hypertension, or infections) because Tac interacts strongly with other drugs metabolized by cytochrome P450 enzymes (1). Therefore, the blood Tac concentrations must be constantly monitored during and after transplantation (2).

High-performance liquid chromatography coupled with mass spectrometry (LC-MS/MS) is the most accurate method to measure the blood Tac concentrations following treatment (3). Because the Tac measurement by LC-MS/MS is both labor intensive and time consuming, several alternative techniques are currently preferred, including the microparticle enzyme-linked immunoassay, enzyme-multiplied immunoassay, chemiluminescent microparticle immunoassay, and antibody-conjugated magnetic immunoassay (ACMIA) method (4-6). An appropriate analytical method is important for therapeutic drug monitoring. The ACMIA method is a monoclonal antibody-based immunoassay that requires no pretreatment, and the procedure is highly automated (4). Therefore, the ACMIA method is widely favored for routine monitoring of immunosuppressive drug concentrations. Many recent studies suggest that endogenous antibodies, such as heterophilic antibodies, rheumatoid factor, and other autoantibodies, may interfere with immunoassays (7).

We herein describe a patient diagnosed with myelodysplastic syndrome (MDS) who underwent allogeneic cord blood transplantation (CBT) with Tac prophylaxis. The ACMIA demonstrated falsely elevated blood Tac concentrations because the patient's plasma components interfered with the ACMIA.

${ }^{1}$ Department of Hematology, Kawasaki Medical School, Japan and ${ }^{2}$ Department of Laboratory Medicine, Kawasaki Medical School, Japan Received for publication November 20, 2013; Accepted for publication May 6, 2014

Correspondence to Dr. Taizo Tasaka, taizo@med.kawasaki-m.ac.jp 


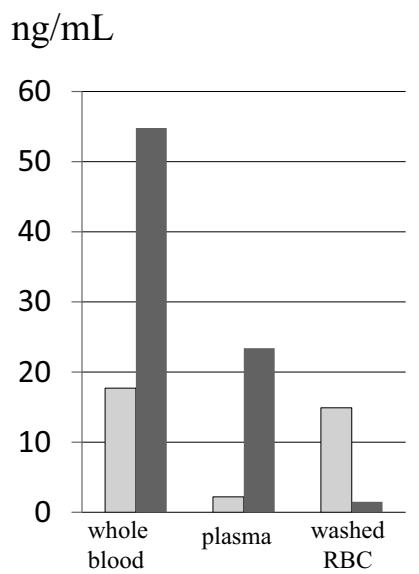

Tac

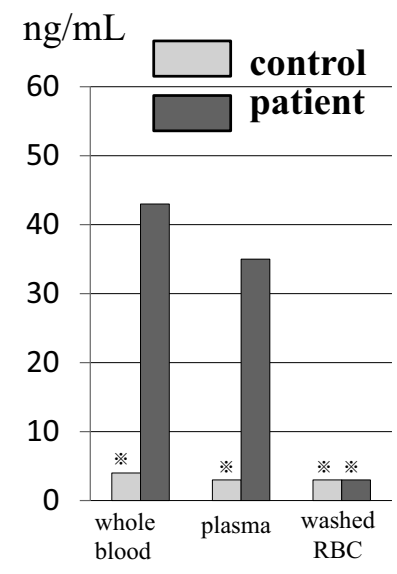

CyA

Figure 1. The effect of plasma deprivation on Tac and CyA concentrations. After washing with saline, the concentration of Tac and CyA in samples was measured by ACMIA. The vertical axis indicates the Tac and $\mathrm{CyA}$ concentrations. The control sample was obtained from another patient who was administrated Tac. ※: below detectable levels

\section{Case Report}

In 2010, a 60-year-old man underwent CBT for MDS [refractory anemia with excess blasts-1, International Prognostic Scoring System (IPSS): int-2]. The patient had a history of intestinal Behçet's disease and had undergone sigmoidectomy two months prior to CBT.

At the time of admission, no abnormal findings were noted during the clinical examination, which included neurological examinations. The conditioning regimen administered before CBT (day 0) consisted of $180 \mathrm{mg} / \mathrm{m}^{2}$ fludarabine, $12.8 \mathrm{mg} / \mathrm{kg}$ busulfan, and $60 \mathrm{mg} / \mathrm{m}^{2}$ melphalan followed by $2.5 \mathrm{mg} / \mathrm{kg}$ anti-thymocyte globulin (ATG). ATG was administered at day -7 and day -6 . The patient received $1.89 \times 10^{7}$ cord blood cells $/ \mathrm{kg}$ body weight. Baseline prophylaxis against GVHD involved Tac and mycophenolate mofetil (MMF). First, Tac $(0.03 \mathrm{mg} / \mathrm{kg})$ was administered intravenously from day -1 to day 27 , then orally after day 27. A daily dose of $1,500 \mathrm{mg}$ MMF was also administered orally from day 3 to day 17 . The blood Tac concentration was routinely measured by the ACMIA method using a Dimension Xpand Plus analyzer (Siemens AG, Berlin, Germany). Successful engraftment was confirmed on day 15 with no sign of acute GVHD during hospitalization.

On day 56, the patient was discharged from our hospital and his hematological parameters suggested a complete recovery. The lymphocyte count was normal $(2,365 / \mu \mathrm{L}$; normal range: $700-3,800 / \mu \mathrm{L}$ ), and the Tac concentration was $9.6 \mathrm{ng} / \mathrm{mL}$. On day 73 , he visited our hospital as scheduled without any symptoms. A routine examination revealed a markedly increased Tac concentration of $23.9 \mathrm{ng} / \mathrm{mL}$. Marked leukocytosis $(16,960 / \mu \mathrm{L})$ and increased lymphocyte subsets $(52.5 \%)$ were also observed. Therefore, the patient

was immediately hospitalized. The Tac therapy was suspended, but the patient remained on steroid monotherapy (10 mg/day prednisolone). Surprisingly, the patient's blood Tac concentration continued to increase, although Tac administration was discontinued. Furthermore, no change was observed in the patient's clinical conditions relative to the Tac discontinuation, including GVHD. On day 81, we decided to verify our measurements of the blood Tac concentration using three methods in parallel. The concentrations obtained by an ACMIA, chemiluminescent immunoassay (CLIA), and enzyme-linked immunosorbent assay (ELISA) were $34.5 \mathrm{ng} / \mathrm{mL}, 2.0 \mathrm{ng} / \mathrm{mL}$, and $<0.5 \mathrm{ng} / \mathrm{mL}$, respectively. These results indicated that the Tac concentrations obtained by ACMIA were falsely elevated.

To determine whether the patient's plasma was the source of interference in the ACMIA method, we measured Tac concentrations in whole blood, plasma, and red blood cells washed with saline (Fig. 1). The concentration of cyclosporine (CyA), which was not administered to the patient, was measured as an internal control. The Tac concentration was $54.8 \mathrm{ng} / \mathrm{mL}$ and the CyA concentration was 43.0 $\mathrm{ng} / \mathrm{mL}$, indicating a false elevation of both components (Fig. 1). Starting on day 111, Tac concentrations were measured by ACMIA in whole blood and plasma-deprived samples (Fig. 2). On day 116, an ELISA and ACMIA detected similar Tac concentrations ( 3.5 vs. $4.3 \mathrm{ng} / \mathrm{mL}$ ) in plasmadeprived samples (Fig. 2). Therefore, Tac administration was resumed at a daily dose of $1.0 \mathrm{mg} / \mathrm{kg}$ (Fig. 2).

\section{Discussion}

The ACMIA is a new, fully automated assay designed to monitor whole blood Tac or CyA concentrations using a mouse capture antibody without any pre-treatment. The benefits of ACMIA are a lighter workload and high throughput (4). Two recent studies reported a high correlation between the whole blood CyA and Tac concentrations measured by ACMIA and alternative immunoassays $(8,9)$. However, our case report indicates that such findings are not always true.

Immunoassays that utilize in vitro immunological binding reactions between the molecule of interest and a reagent antibody may lack monospecificity (10). Our case showed that ACMIA yielded falsely elevated whole blood Tac and CyA concentrations in a patient with MDS who received CBT. Previous studies have also reported falsely elevated Tac and CyA measurements due to immunological assay interferences $(4,9-11)$. It has been suggested that endogenous heterophilic antibodies or human antibodies binding to animal antibodies used in certain immunoassays may interfere with the immunological assays and lead to erroneous results. These heterophilic antibodies are produced in response to infection, recent immunization, treatment with monoclonal antibodies, blood transfusion, exposure to animal proteins, or autoimmune disorders (10). In fact, falsely elevated Tac concentrations measured by ACMIA were reported in 2 of 


\section{Tac dose}

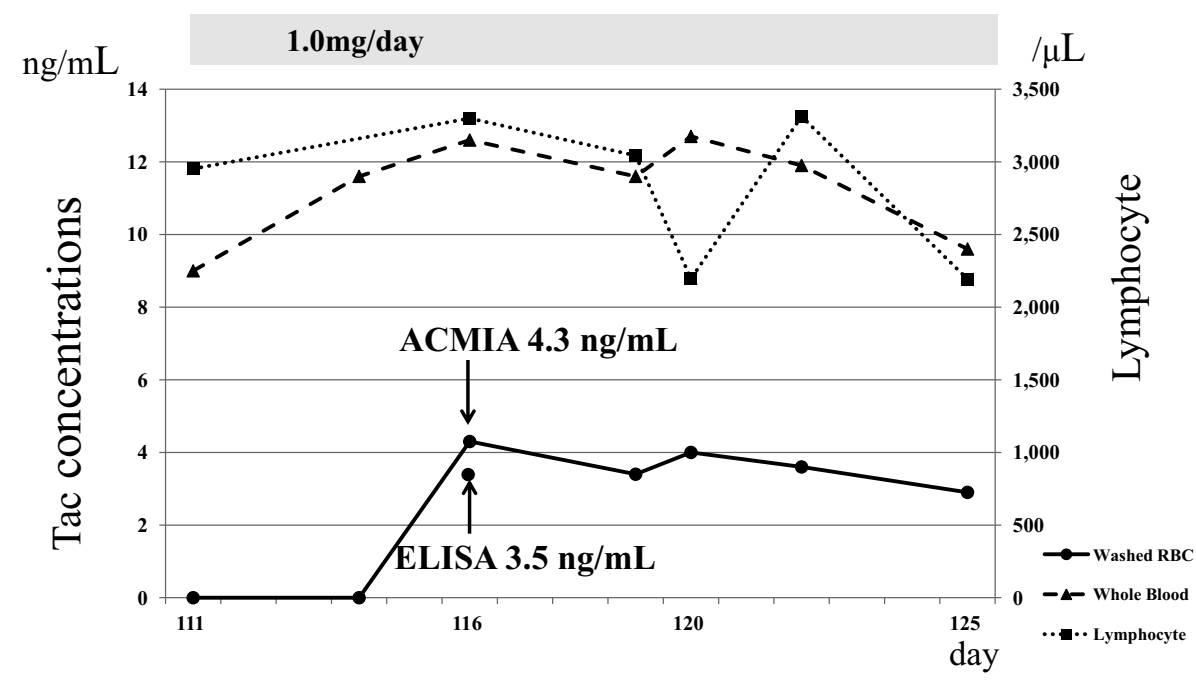

Figure 2. Tac concentrations after resuming its administration. The vertical axis indicates the Tac concentrations. The horizontal axis indicates the days after transplantation. The dashed line indicates the Tac concentrations in the whole blood. The solid line indicates the Tac concentrations in the plasma-deprived (washed with saline) samples. The dotted line indicates lymphocyte numbers. ELISA: enzyme-linked immunosorbent assay

$50(4 \%)$ patients seropositive for rheumatoid arthritis but not given Tac.

Previous reports have demonstrated that such a phenomenon may be related to autoimmune diseases (12). Our patient had a history of Behçet's disease, which may have affected his immune status. In addition, the use of rabbit ATG in transplant recipients is associated with the development of serum anti-rabbit antibodies (13), potentially leading to clinically significant interferences, as previously reported (14). Falsely elevated Tac concentrations were not observed immediately after ATG administration but at day 73 after CBT. This timeline suggests that ATG may suppress immunocompetent cells. Studies have analyzed immune reconstitution after ATG-conditioned HSCT. The recovery of $\mathrm{B}$ cells and memory/effector CD8 $\mathrm{T}$ cells occur after the second and third months of ATG conditioning, which is more rapid than with non-ATG conditioning (15). We detected a marked increase in the lymphocyte count from $2,365 / \mu \mathrm{L}$ on day 56 to $8,900 / \mu \mathrm{L}$ on day 73 while the patient was discharged from the hospital. On day 59, 26\% of the lymphocytes were B cells (data not shown), thus suggesting a certain level of immune reconstitution at this point. In addition, after Tac was resumed starting on day 111, the lymphocyte count gradually decreased, as well as the falsely elevated Tac concentration, suggesting a relationship between the interference and immune reconstitution after ATG administration. Therefore, ATG-conditioned HSCT may cause the transient production of interfering molecules, such as heterophilic antibodies. The etiology of these interferences during immunoassays remains incomplete. However, falsely elevated CyA concentrations were reported in a patient diagnosed with familial hemophagocytic lymphohistiocytosis who was receiving CBT, and this artefact first ap- peared two months after transplantation (16).

The exact cause of this phenomenon remains unclear, but our findings indicate that such interference can be easily avoided. More than $95 \%$ of Tac is sequestered in the erythrocyte fraction of whole blood, and $<2 \%$ is found in the plasma (17). Therefore, Tac should only be detected at low concentrations in control plasma. In our patient, plasma Tac concentrations were high, suggesting that the interfering molecules, such as heterophilic antibodies, are in found plasma. We removed all plasma from the sample by centrifugal separationand results for the Tac concentration measurement by ACMIA were similar to those by ELISA (Fig. 2). A simple pre-treatment with heterophilic blocking tubes was also shown to prevent false high Tac concentrations using the ACMIA method (12).

Based on our clinical experience and the literature review currently available on Tac assays, we caution clinicians regarding we do not recommend the use of the ACMIA method to monitor blood Tac concentrations in patients with a history of autoimmune disease and/or receiving xenogeneic antibody therapy without proper specimen preparation.

The authors state that they have no Conflict of Interest (COI).

\section{References}

1. Holt DW, Armstrong VW, Griesmacher A, Morris RG, Napoli KL, Shaw LM; International Federation of Clinical Chemistry, International Association of Therapeutic Drug Monitoring, Clinical Toxicology working group on immunosuppressive drug monitoring. International Federation of Clinical Chemistry/International Association of Therapeutic Drug Monitoring and Clinical Toxicology working group on immunosuppressive drug monitoring. Ther Drug Monit 24: 59-67, 2002. 
2. Christians U, Jacobsen W, Benet LZ, Lampen A. Mechanisms of clinically relevant drug interactions associated with tacrolimus. Clin Pharmacokinet 41: 813-851, 2002.

3. Koster RA, Dijkers EC, Uges DR. Robust, high-throughput LCMS/MS method for therapeutic drug monitoring of cyclosporine, tacrolimus, everolimus, and sirolimus in whole blood. Ther Drug Monit 31: 116-125, 2009.

4. Ju MK, Chang HK, Kim HJ, et al. Is the affinity column-mediated immunoassay method suitable as an alternative to the microparticle enzyme immunoassay method as a blood tacrolimus assay? Transplant Proc 40: 3673-3678, 2008.

5. Bartlomiejczyk I, Zochowska D, Sanko-Resmer J, Matuszewicz D, Paczek L. Therapeutic monitoring of tacrolimus concentrations in blood of renal and liver transplant recipients: comparison of microparticle enzyme immunoassay and enzyme multiplied immunoassay methods. Transplant Proc 38: 94-96, 2006.

6. Hétu PO, Robitaille R, Vinet B. Successful and cost-efficient replacement of immunoassays by tandem mass spectrometry for the quantification of immunosuppressants in the clinical laboratory. Chromatogr B Analyt Technol Biomed Life Sci 883-884: 95-101, 2012.

7. D’Alessandro M, Mariani P, Mennini G, Severi D, Berloco P, Bachetoni A. Falsely elevated tacrolimus concentrations measured using the ACMIA method due to circulating endogenous antibodies in a kidney transplant recipient. Clin Chim Acta 412: 245-248, 2011.

8. Moscato D, Nonnato A, Adamo R, Vancheri M, Caropreso A. Therapeutic monitoring of tacrolimus: aberrant results by an immunoassay with automated pretreatment. Clin Chim Acta 411: 7780, 2010.

9. Huet E, Morand K, Blanchet B, Astier A, Hulin A. Evaluation of the new heterogeneous ACMIA immunoassay for the determination of whole-blood cyclosporine concentrations in bone marrow, kidney, heart, and liver transplant recipients. Transplant Proc 36: 1317-1320, 2004.

10. IsmaiJ Y, lsmail AA, lsmail AA. Erroneous laboratory results: what clinicians need to know. Clin Med 7: 357-361, 2007.

11. Toraishi $T$, Takeuchi $H$, Nakamura $Y$, et al. Falsely abnormally elevated blood trough concentration of tacrolimus measured by antibody-conjugated magnetic immunoassay in a renal transplant recipient: a case report. Transplant Proc 44: 134-136, 2012.

12. Barceló Martín B, Marquet P, Ferrer JM, et al. Rheumatoid factor interference in a tacrolimus immunoassay. Ther Drug Monit 31: 743-745, 2009.

13. Prin Mathieu C, Renoult E, Kennel De March A, et al. Serum anti-rabbit and anti-horse $\operatorname{IgG}, \operatorname{IgA}$, and $\operatorname{IgM}$ in kidney transplant recipients. Nephrol Dial Transplant 12: 2133-2139, 1997.

14. Benoist JF, Orbach D, Biou D. False increase in C-reactive protein attributable to heterophilic antibodies in two renal transplant patients treated with rabbit antilymphocyte globulin. Clin Chem 44: 1980-1985, 1998.

15. Bosch M, Dhadda M, Hoegh-Petersen M, et al. Immune reconstitution after antithymocyte globulin conditioned hematopoietic cell transplantation. Cytotherapy 14: 1258-1275, 2012.

16. Bartoli A, Molinaro M, Visai L. Falsely elevated whole blood cyclosporine concentrations measured by an immunoassay with automated pretreatment. Ther Drug Monit 32: 791-792, 2010.

17. Nagase K, Iwasaki K, Nozaki K, Noda KA. Distribution and protein binding of FK506, a potent immunosuppressive macrolide lactone, in human blood and its uptake by erythrocytes. J Pharm Pharmacol 46: 113-117, 1994.

(C) 2014 The Japanese Society of Internal Medicine http://www.naika.or.jp/imonline/index.html 